\title{
Changes in Sympathetic Nervous System Activity in Male Smokers After Moderate-Intensity Exercise
}

\author{
Hiroshi Ide PT MSc and Kazuyuki Tabira PT PhD
}

\begin{abstract}
OBJECTIVE: To investigate the effects of moderate-intensity exercise on the sympathetic nervous system in male smokers. METHODS: Twenty-eight men (14 smokers and 14 non-smokers, ages 21-46 y) were recruited. The activity of the autonomic nervous system was measured by power spectral analysis of heart rate variability. Spectral power in the frequency domain was quantified by integrating the area under the curve of the very-low-frequency power $(0.007-0.035 \mathrm{~Hz})$, lowfrequency power $(0.035-0.15 \mathrm{~Hz})$, high-frequency power $(0.15-0.5 \mathrm{~Hz})$, and total power $(0.007-$ $0.5 \mathrm{~Hz}$ ) bandwidths. We assessed heart rate, thermoregulatory sympathetic nervous system activity (very-low-frequency power/total power), sympathetic nervous system activity (low-frequency-power/high-frequency power or [very-low-frequency power + low-frequency power]/high-frequency power), and parasympathetic nervous system activity (high-frequency power/total power) of the smokers before and after moderate-intensity exercise. RESULTS: The smokers exhibited a greater degree of sympathetic nervous system activity (as quantified by very-low-frequency power/total power). The sympathetic nervous system activity of smokers (as indicated by [very-low-frequency power + low-frequency power]/high-frequency power) also showed a tendency to increase after exercise. Parasympathetic activity, as indicated by high-frequency power/total power, reduced after exercise in the smokers. These findings are contrary to findings previously reported in obese subjects. CONCLUSIONS: Increased sympathetic nervous system activity, including thermoregulatory activity, might contribute to cachexia in smokers. Key words: smoker; exercise; heart rate variability; autonomic responsiveness; power spectral analysis; very-low-frequency power; sympathetic nervous system activity; cachexia. [Respir Care 2013;58(11):1892-1898. () 2013 Daedalus Enterprises]
\end{abstract}

\section{Introduction}

Cachexia is a complex metabolic syndrome characterized by loss of muscle, with or without loss of fat mass, and is most often associated with underlying illness. ${ }^{1}$ Many

Mr Ide and Dr Tabira are affiliated with the Division of Health Science, Graduate School of Health Science, Kio University, Koryo, Nara, Japan. Mr Ide is also affiliated with the Department of Physical Medicine and Rehabilitation, Tsu Seikyou Hospital, Tsu, Mie, Japan.

This study was supported by joint research grant H22-16 from Kio University, Nara, Japan. The authors have disclosed no conflicts of interest.

Correspondence: Hiroshi Ide PT MSc, Department of Physical Medicine and Rehabilitation, Tsu Seikyou Hospital, 1721 Sendoumachi, Tsu City Mie, Japan. E-mail: h_idet@yahoo.co.jp.

DOI: $10.4187 /$ respcare. 02240 patients with chronic and end-stage diseases, including infection, cancer, and congestive heart failure, demonstrate nutritional changes characteristic of cachexia. ${ }^{1,2}$ Patients in the early stages of COPD also display systemic features such as reduced fat mass, ${ }^{3}$ muscle loss, and skeletal muscle wasting. ${ }^{4,5}$ The pathogenesis of these features is complex and may result from multiple factors, including energy imbalance, disuse atrophy, and systemic inflammation. ${ }^{6}$ Furthermore, cigarette smoking, which is a major risk factor for COPD, cancer, and congestive heart failure, ${ }^{7}$ has been linked to reductions in body weight and body mass index. ${ }^{8}$ These effects are thought to be due to increased energy expenditure and a reduction in appetite. ${ }^{8}$ Thus, cigarette smoking may be associated with weight loss even before the development for COPD or other pathologies.

Energy expenditure antagonizes the activity of the autonomic nervous system, which controls the heart rate and rhythm. The R-R interval, also known as the inter-beat interval, is determined by the net effect of sympathetic and 
parasympathetic inputs, and can be measured via electrocardiogram. Spectral analysis of heart-rate variability has gained general acceptance as an indicator of autonomic nervous system function. ${ }^{9}$ Power spectral analysis of heartrate variability has demonstrated at least 2 distinct regions of periodicity within the electrocardiogram R-R interval: high-frequency power $(>0.15 \mathrm{~Hz})$, which solely reflects parasympathetic nervous system activity, and lowfrequency power $(<0.15 \mathrm{~Hz})$, which is dually mediated by sympathetic nervous system and parasympathetic nervous system activity. ${ }^{9}$

Previous studies using power spectral analysis have demonstrated that inactivity of the autonomic nervous system, particularly the sympathetic nervous system, may indicate a risk of future weight gain and development of obesity. ${ }^{10}$ Furthermore, recent obesity studies have suggested that very-low-frequency power $(0.007-0.035 \mathrm{~Hz})$ of heart-rate variability is selectively lowered in obese individuals, in response to several thermogenic perturbations such as acute cold exposure and food intake. ${ }^{11,12}$ That research demonstrated that spectral analysis of very-low-frequency power of heart-rate variability is a means of evaluating energy metabolism and thermoregulatory sympathetic nervous system function in humans.

To our knowledge, the effect of moderate-intensity exercise on the very-low-frequency power of heart-rate variability has not previously been investigated in smokers. The primary objective of this study was to evaluate, via power spectral analysis of heart-rate variability, the verylow-frequency activity of heart-rate variability in smokers and non-smokers after moderate-intensity exercise. We hypothesized that smokers would display continual hyperactive very-low-frequency power of heart-rate variability.

\section{Methods}

The ethics board of Kio University and the hospital ethics committee of Tsu Seikyou Hospital approved the study protocol. Written informed consent was obtained from all subjects.

\section{Subjects}

Height- and weight-matched male non-smokers $(n=14)$ and smokers $(n=14)$ between the ages of 21 and 46 years were recruited for the study. In previous studies, autonomic nervous system activity was found to vary with body type. ${ }^{10-12}$ Therefore, we matched body types among the subjects to isolate the effects of cigarette smoking. The smokers were asked about the duration and frequency of their smoking. The weight and fat-free mass of each subject were measured with a bioelectrical impedance analyzer (InnerScan 50V, Tanita, Tokyo, Japan). Body mass index and fat-free mass index were calculated as body

\section{QUICK LOOK}

\section{Current knowledge}

Cachexia is a complex metabolic syndrome characterized by muscle loss, with or without loss of fat mass, and is associated with underlying illnesses such as COPD.

\section{What this paper contributes to our knowledge}

Smokers had a greater degree of sympathetic nervous system activity than non-smokers. Increased sympathetic nervous system activity, including thermoregulatory activity, may contribute to cachexia in smokers.

weight/height ${ }^{2}$ and fat-free mass/height ${ }^{2}$, respectively. Subject demographics are presented in Table 1. Members of both the smoking and non-smoking groups were in good health and had no evidence of hypertension, cardiovascular disease, diabetes mellitus, or other disease. Every subject kept regular hours at the university and/or at jobs. None of the subjects reported engaging in regular exercise. Each subject was instructed to avoid alcohol, coffee, and tea, and to fast for 8 hours preceding the study, to reduce the effects of alcohol, caffeine, and food on the autonomic nervous system. None of the subjects reported a history of heavy drinking, a habit of staying up late, or engaging in extreme exercise the day before the study. Subjects who smoked were asked to abstain from smoking for 8 hours before the study, to limit the acute effects of smoking, such as increased heart rate, on the measurements. ${ }^{13,14}$ The continuous exercise load used in this study was determined using the Karvonen formula, so that the increased heart rate would place the same load on the bodies of smokers and non-smokers. On the day of the study the subjects stayed in a quiet, comfortable room with minimal stimuli from 2:00 PM to 6:00 PM. This period of the day was chosen to reduce the effects of the circadian rhythm on autonomic nervous system activity. In accordance with previous studies, ${ }^{11,12}$ the temperature and humidity of the room were strictly regulated (see Table 1), and all subjects were dressed uniformly in T-shirts and shorts, made of the same material.

\section{Experimental Procedures}

Subjects were seated in comfortable chairs and allowed to rest for at least $20 \mathrm{~min}$ before the start of the experiment. Each subject breathed in time with a metronome set at 15 beats/min, to ensure that respiratory-linked variations in heart rate did not interfere with low-frequency $(<0.15 \mathrm{~Hz})$ heart rate fluctuations from other sources. 
Table 1. Subject Demographics

\begin{tabular}{lcc}
\hline \hline & $\begin{array}{c}\text { Non-Smokers } \\
n=14\end{array}$ & $\begin{array}{c}\text { Smokers } \\
n=14\end{array}$ \\
\hline Age, $\mathrm{y}$ & $28.3 \pm 7.2$ & $27.6 \pm 6.8$ \\
Height, $\mathrm{cm}$ & $168.3 \pm 6.9$ & $170.9 \pm 6.4$ \\
Weight, $\mathrm{kg}$ & $65.43 \pm 9.98$ & $63.65 \pm 8.95$ \\
Body mass index, $\mathrm{kg} / \mathrm{m}^{2}$ & $23.0 \pm 2.5$ & $21.7 \pm 2.8$ \\
Fat-free mass, $\mathrm{kg}$ & $50.51 \pm 6.37$ & $49.26 \pm 5.09$ \\
Fat-free mass index, $\mathrm{kg} / \mathrm{m}^{2}$ & $17.8 \pm 1.4$ & $16.8 \pm 1.3$ \\
Room temperature, ${ }^{\circ} \mathrm{C}$ & $24.4 \pm 1.5$ & $24.0 \pm 0.7$ \\
Humidity, $\%$ & $45.6 \pm 4.3$ & $44.3 \pm 1.6$ \\
Smoking history & & \\
$\quad$ Duration, $\mathrm{y}$ & & $8.8 \pm 6.5$ \\
$\quad$ Cigarettes per day & & $10.8 \pm 7.5$ \\
$\quad$ Pack-years & & $6.3 \pm 9.9$ \\
Blood pressure, mm $\mathrm{Hg}$ & & \\
$\quad$ Systolic & & \\
$\quad$ Diastolic & $121.3 \pm 7.1$ & $69.7 \pm 6.7$ \\
& & \\
Values are mean $\pm \mathrm{SD}$. & & \\
Blood pressure was measured before exercise. \\
The only significant differences between the groups were in duration of cigarette smoking, \\
cigarettes per day, and pack-years.
\end{tabular}

The breathing frequency was regulated for 10 min before the exercise commenced, and during the recovery period. The regulation of respiration prevented placing a substantial load on the subject during the exercise period. The recovery period lasted for $30 \mathrm{~min}$ after exercise. The target heart rate for the continuous exercise load was set using the Karvonen formula:

$$
\begin{aligned}
& \text { (Maximum heart rate }- \text { at-rest heart rate) } \\
& \quad \times 60 \% \text { intensity }+ \text { at-rest heart rate }
\end{aligned}
$$

The intensity level was set at $60 \%$ of the estimated maximal heart rate, which was calculated as (220 - age). Moderate-intensity exercise was performed on a bicycle ergometer (Combi, Tokyo, Japan), and consisted of pedaling at 60 revolutions $/ \mathrm{min}$. The initial power output was $25 \mathrm{~W}$. The subjects achieved $60 \%$ intensity within $5 \mathrm{~min}$, and the exercise was continued at this intensity for another 15 min. Heart rate was continuously measured using a sports heart rate meter (S801i, Polar Electro, Kempele, Finland).

\section{R-R Interval Power Spectral Analysis}

Periodic components of heart-rate variability tend to aggregate within several frequency bands. ${ }^{9}$ Autonomic nervous system activity can therefore be measured noninvasively by heart-rate variability power spectral analysis, which deconstructs a series of sequential R-R intervals into a sum of sinusoidal functions of different amplitudes and frequencies, via the Fourier transform algorithm. In the present investigation, 3 frequency components of heartrate variability were detected and analyzed. This approach has been used in basic medical science to assess a diverse range of conditions, and its validity and reliability have been confirmed in the literature. ${ }^{11,12}$ The spectral powers in frequency domains were quantified by integrating the area under the curve for the following bandwidths: verylow-frequency power $(0.007-0.035 \mathrm{~Hz})$, reflecting energy metabolic and thermoregulatory sympathetic function; lowfrequency power $(0.035-0.15 \mathrm{~Hz})$, reflecting both sympathetic nervous system and parasympathetic nervous system activity; high-frequency power $(0.15-0.5 \mathrm{~Hz})$, which solely reflects parasympathetic nervous system activity; and total power $(0.007-0.5 \mathrm{~Hz})$, which represents overall autonomic nervous system activity. ${ }^{11,12}$ Thermoregulatory sympathetic nervous system activity was calculated as verylow-frequency/total. Sympathetic nervous system activities was calculated as low-frequency/high-frequency and (very-low-frequency + low-frequency)/high-frequency ratio. Parasympathetic nervous system activity was calculated as high-frequency/total.

The pre-exercise rest period was $5 \mathrm{~min}$, and the postexercise recovery period was $30 \mathrm{~min}$, which we analyzed in 5-min stages: 5-10 min post-exercise, $10-15$ min postexercise, 15-20 min post-exercise, 20-25 min post-exercise, and 25-30 min post-exercise. The segments provide a direct view of time-series changes in autonomic nervous system activity. We did not analyze data from the 5-min period immediately following exercise.

\section{Statistical Analysis}

Data analysis was performed using statistics software (SPSS 19.0, SPSS Chicago, Illinois). Differences between groups were analyzed by comparing the heart rate, verylow-frequency/total, low-frequency/high-frequency, (verylow-frequency + low-frequency)/high-frequency, and high-frequency/total for the 6 time periods. The data distribution was analyzed with the Kolmogorov-Smirnov test. The heart rate, very-low-frequency/total, low-frequency/ high-frequency, (very-low-frequency + low-frequency)/ high-frequency, and high-frequency/total values were compared with the Mann-Whitney U test, because these data were not normally distribution. The results of these analyses were consistent in all cases. Data are expressed as median (minimum-maximum). Statistical significance was defined as $P<.05$.

\section{Results}

Figure 1 and Table 2 show the heart rate and power values. The very-low-frequency/total values were signifi- 

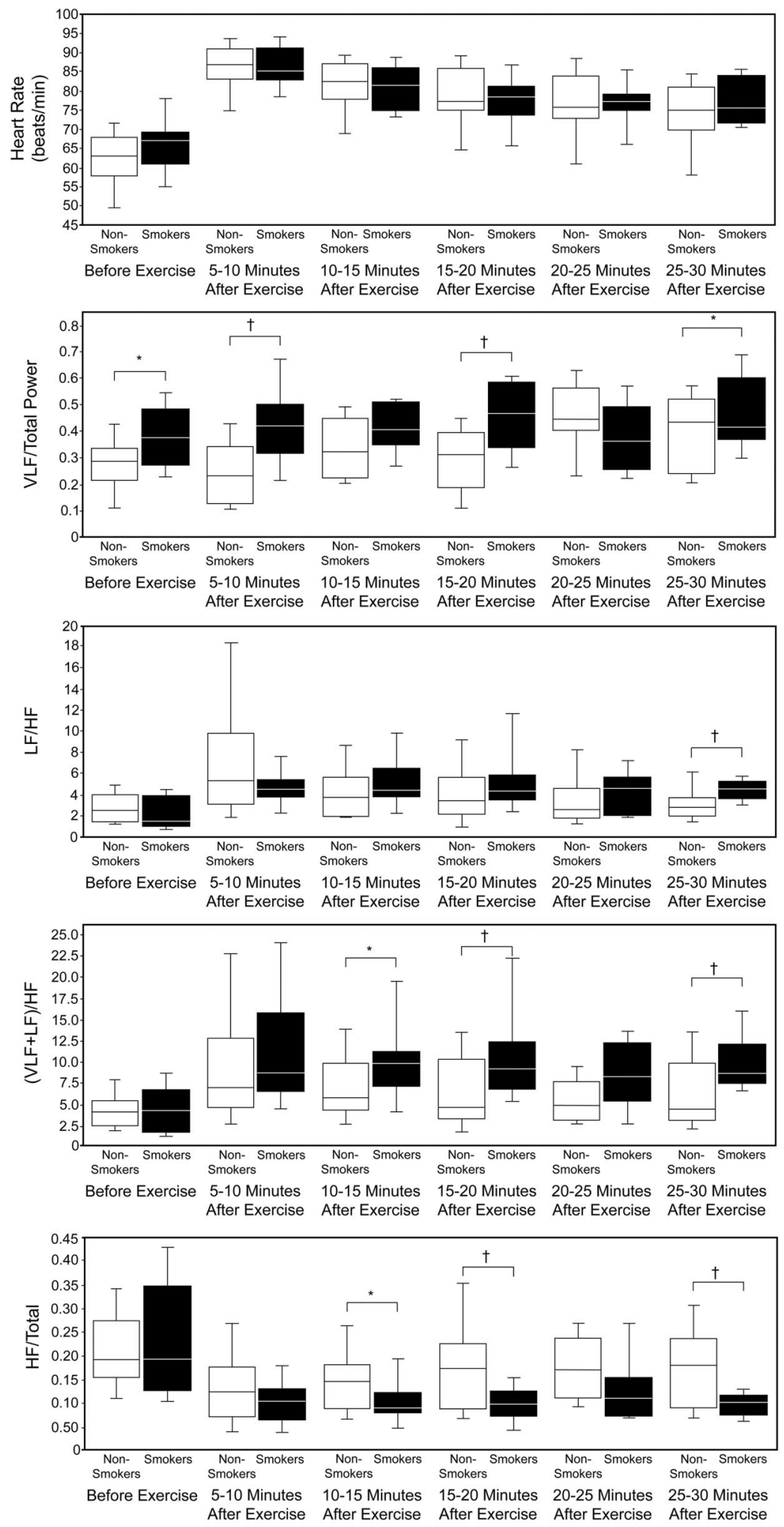

Fig. 1. Heart rate, ratio of the very-low-frequency (VLF) power component to the total power, ratio of the low-frequency (LF) power component to the high-frequency (HF) power component, ratio of (VLF + LF) to the HF power component, and ratio of the HF power component to total power. In the data bars the horizontal line represents the median, the tops and bottoms of the bars represent the first and third quartiles, and the whisker bars represent the 10th and 90th percentiles. The test periods were analyzed with the Mann-Whitney $\mathrm{U}$ test: ${ }^{*} P<.10, \dagger P<.05$. 
Table 2. Comparison of Combined Heart Rate Variability in Non-Smokers and Smokers Over the Course of 6 Time-Periods

\begin{tabular}{|c|c|c|c|}
\hline & $\begin{array}{c}\text { Non-Smokers } \\
\text { Median (min-max) }\end{array}$ & $\begin{array}{c}\text { Smokers } \\
\text { Median (min-max) }\end{array}$ & $P^{*}$ \\
\hline \multicolumn{4}{|l|}{ Heart rate, beats/min } \\
\hline Before exercise & $63.0(47-76)$ & $66.5(50-79)$ & .32 \\
\hline 5-10 min after exercise & $87.0(66-110)$ & $85.0(75-98)$ & .96 \\
\hline 10-15 min after exercise & $85.5(60-103)$ & $81.5(68-96)$ & .78 \\
\hline 15-20 min after exercise & $77.5(53-101)$ & $78.5(66-93)$ & .98 \\
\hline 20-25 min after exercise & $76.0(53-94)$ & $77.0(58-91)$ & .73 \\
\hline 25-30 min after exercise & $75.0(50-99)$ & $75.5(70-92)$ & .55 \\
\hline \multicolumn{4}{|l|}{ Low-frequency/high-frequency } \\
\hline Before exercise & $2.55(0.87-6.71)$ & $1.57(0.60-6.47)$ & .36 \\
\hline 5-10 min after exercise & $5.28(1.73-18.58)$ & $4.62(1.87-17.10)$ & .61 \\
\hline 10-15 min after exercise & $3.78(1.65-10.50)$ & $5.22(2.25-12.38)$ & .12 \\
\hline 15-20 min after exercise & $3.44(0.88-11.10)$ & $4.37(2.35-12.42)$ & .18 \\
\hline 20-25 min after exercise & $2.64(1.21-8.62)$ & $4.67(1.72-10.92)$ & .15 \\
\hline 25-30 min after exercise & $2.80(1.46-10.76)$ & $4.70(2.77-9.26)$ & .01 \\
\hline \multicolumn{4}{|l|}{ High-frequency/total } \\
\hline Before exercise & $0.19(0.09-0.36)$ & $0.20(0.06-0.43)$ & .78 \\
\hline 5-10 min after exercise & $0.12(0.03-0.30)$ & $0.10(0.03-0.18)$ & .25 \\
\hline 10-15 min after exercise & $0.15(0.07-0.32)$ & $0.09(0.05-0.22)$ & .09 \\
\hline 15-20 min after exercise & $0.18(0.05-0.39)$ & $0.10(0.31-0.18)$ & .02 \\
\hline 20-25 min after exercise & $0.17(0.08-0.28)$ & $0.11(0.04-0.29)$ & .12 \\
\hline 25-30 min after exercise & $0.18(0.05-0.31)$ & $0.10(0.03-0.13)$ & .048 \\
\hline \multicolumn{4}{|l|}{ Very-low-frequency/total } \\
\hline Before exercise & $0.29(0.09-0.62)$ & $0.38(0.22-0.56)$ & .09 \\
\hline 5-10 min after exercise & $0.24(0.05-0.50)$ & $0.43(0.18-0.86)$ & .005 \\
\hline $10-15$ min after exercise & $0.33(0.16-0.58)$ & $0.41(0.10-0.55)$ & .13 \\
\hline 15-20 min after exercise & $0.31(0.10-0.48)$ & $0.47(0.18-0.63)$ & .005 \\
\hline 20-25 min after exercise & $0.36(0.12-0.56)$ & $0.37(0.21-0.64)$ & .46 \\
\hline 25-30 min after exercise & $0.28(0.07-0.68)$ & $0.42(0.29-0.70)$ & .054 \\
\hline \multicolumn{4}{|c|}{$($ Very-low-frequency + low-frequency)/high-frequency } \\
\hline Before exercise & $4.23(1.82-9.72)$ & $4.14(1.30-15.47)$ & .78 \\
\hline 5-10 min after exercise & $7.10(2.31-37.18)$ & $8.72(4.45-35.07)$ & .25 \\
\hline $10-15$ min after exercise & $5.89(2.18-14.03)$ & $9.82(3.58-19.54)$ & .09 \\
\hline 15-20 min after exercise & 4.66 (1.55-17.90) & $9.10(4.65-31.30)$ & .02 \\
\hline 20-25 min after exercise & $4.90(2.54-11.00)$ & $8.19(2.45-22.50)$ & .12 \\
\hline 25-30 min after exercise & $4.55(2.26-19.73)$ & $8.64(6.61-33.22)$ & .048 \\
\hline
\end{tabular}

cantly higher in the smokers during the 5-10-min postexercise period $(P=.005)$ and the $15-20$-min post-exercise period $(P=.005)$. The low-frequency/high-frequency value was significantly higher in the smokers during the 25-30-min post-exercise period $(P=.01)$. The (very-lowfrequency + low-frequency)/high-frequency values were significantly higher in the smokers during the 15-20-min post-exercise period $(P=.02)$ and the $25-30$-min postexercise period $(P=.048)$. The high-frequency/total value was significantly lower in the smokers in the 15-20-min post-exercise period $(P=.02)$ and the $25-30$-min postexercise period $(P=.048)$. The very-low-frequency/total value in the pre-exercise period and the 25-30-min post-exercise period, and the (very-low-frequency + low- frequency)/high-frequency value in the $10-15$-min postexercise period were higher in the smokers. And the highfrequency/total value in the $10-15$-min post-exercise period was lower in the smokers. None of the other measured parameters differed significantly.

\section{Discussion}

After moderate-intensity exercise, the very-low-frequency/ total and (very-low-frequency + low-frequency)/highfrequency values were higher, and the high-frequency/total value was lower in the smokers than in non-smokers with identical exercise loads. In addition, the very-lowfrequency/total value before exercise and the low-frequen- 
cy/high-frequency value were higher in the smokers. These findings are contrary to those previously reported for obese subjects. ${ }^{11,12}$ Exercise in smokers may thus induce excessive increases in sympathetic nervous system activity, including thermoregulatory sympathetic nervous system activity.

The very-low-frequency activity of thermoregulatory sympathetic function reflects lipid metabolism. In obese individuals, very-low-frequency activity does not increase after thermogenic perturbations, ${ }^{11,12}$ and can be altered through dietary modification and exercise training. ${ }^{15,16}$ Very-low-frequency activity is associated with mutations in uncoupling proteins and $\beta_{3}$-adrenergic receptors within adipose tissue. ${ }^{17,18}$ The activation of uncoupling proteins 1 , 2 , and 3, which are located in white adipose tissue, brown adipose tissue, and skeletal muscle, respectively, converts energy from the substrate into heat without using adenosine triphosphate. ${ }^{19}$ Thermoregulatory sympathetic nervous system activity significantly correlates with plasma leptin concentration. ${ }^{20}$ Thus, our results suggest that the elevated very-low-frequency/total in smokers reflects increased thermoregulatory sympathetic nervous system activity, both before and after exercise, which may over-stimulate the catabolism of fat in adipose tissue.

The changes we observed in very-low-frequency/total, low-frequency/high-frequency, (very-low-frequency + low-frequency)/high-frequency, and high-frequency/total in smokers persisted for $30 \mathrm{~min}$ after exercise. These changes affect the sympathetic nervous system via metaboreflex, baroreflex, and central command. ${ }^{21}$ In a previous study, smokers had higher resting energy expenditure, mediated by caffeine as a thermogenic perturbation. ${ }^{22}$ Caffeine and nicotine during casual physical activity increase energy expenditure in smokers. ${ }^{23}$ Analysis of expired gas has revealed that the total thermic response of smokers is significantly greater than that of non-smokers over a period of 4-24 hours. ${ }^{24,25}$ Normal-weight smokers have a higher energy expenditure at rest for $30 \mathrm{~min}$ after smoking than do obese smokers. ${ }^{26}$ Therefore, the already over-activated sympathetic nervous system in normalweight smokers may be further activated in very-lowfrequency/total, low-frequency/high-frequency, and (verylow-frequency + low-frequency)/high-frequency by exercise.

These findings are clinically important. We deduce that the sympathetic nervous system of smokers may mobilize against adipose tissue, triggering cachexia when calorie intake is deficient. Elevation of the hypothalamic-sympathetic nervous system by leptin occurs indirectly, through oxidation of fatty acids in muscle. ${ }^{27}$ Smokers also experience muscle wasting ${ }^{28}$ and loss of type- 1 muscle fibers. ${ }^{29}$ Furthermore, weight loss, including cachexia, is associated with increased morbidity and mortality. $5,30,31$ Thus, nor- mal-weight smokers may benefit from anti-smoking measures to prevent weight loss.

These changes in autonomic nervous system activity are presumably due to the action of nicotine. Subjects in this study abstained from cigarette smoking for 8 hours to limit the acute effects of cigarette smoking. No significant differences were observed in the heart rate or low-frequency/ high-frequency values between smokers and nonsmokers during any of the 6 time periods. This observation demonstrates that the acute effects of cigarette smoking were absent in these subjects. However, the effects of nicotine persist for at least 6 hours after cessation of smoking. ${ }^{14}$ Nicotine activates sympathetic neurotransmission by inducing the release of ganglion catecholamine from postganglionic nerve endings. ${ }^{32}$ In the present study, the lowfrequency/high-frequency value in the 5-10-min postexercise period was lower in the smokers, with an increase in the very-low-frequency/total value. The sympathetic nervous system activity (very-low-frequency/total and [verylow-frequency + low-frequency]/high-frequency) of smokers remained elevated throughout the post-exercise period. Thus, the thermoregulatory sympathetic nervous system value in smokers may be more sensitive to nicotine than the low-frequency/high-frequency value.

\section{Limitations}

First, we did not conduct all the tests at the same room temperature and humidity. Temperature and humidity do play a role in autonomic nervous system activity, so inconsistencies may be present in the results. Second, we did not directly measure lipid metabolism and cannot conclusively say that thermoregulatory sympathetic nervous system activity corresponds to lipid metabolism. To compensate, we used only healthy subjects with normal weight. Future studies should include subjects with cachexia, semistarvation condition, or muscle atrophy. Additionally, metabolic and nutritional studies are required to confirm the relationship between sympathetic nervous system activity, body weight, and metabolic profiles.

\section{Conclusions}

Exercise induced hyperactivity of the sympathetic nervous system (thermoregulatory sympathetic nervous system and [very-low-frequency + low-frequency]/high-frequency) in smokers. Furthermore, the high-frequency/total value did not return to the pre-exercise level after exercise in smokers. This sympathetic nervous system hyperactivity in smokers may over-stimulate fat catabolism in adipose tissue over an extended period and trigger cachexia.

\section{ACKNOWLEDGMENTS}

We are grateful to members of the Tabira laboratory for their helpful suggestions. 


\section{REFERENCES}

1. Evans WJ, Morley JE, Argilés J, Bales C, Baracos V, Guttridge D, et al. Cachexia: a new definition. Clin Nutr 2008;27(6):793-799.

2. Kotler DP. Cachexia. Ann Intern Med 2000;133(8):622-634.

3. Schols AM, Slangen J, Volovics L, Wouters EF. Weight loss is a reversible factor in the prognosis of chronic obstructive pulmonary disease. Am J Respir Crit Care Med 1998;157(6):1791-1797.

4. Marquis K, Deblgare R, Lacasse Y, LeBlanc P, Jobin J, Carrier G, et al. Midthigh muscle cross-sectional area is a better predictor of mortality than body mass index in patients with chronic obstructive pulmonary disease. Am J Respir Crit Care Med 2002;166(6):809813.

5. Schols AM, Broekhuizen R, Weling-Scheepers CA, Wouters EF. Body composition and mortality in chronic obstructive pulmonary disease. Am J Clin Nutr 2005;82(1):53-59.

6. Wagner PD. Possible mechanisms underlying the development of cachexia in COPD. Eur Respir J 2008;31(3):492-501.

7. American Thoracic Society. Cigarette smoking and health. Am J Respir Crit Med 1996;153(2):861-865.

8. Chiolero A, Faeh D, Paccaud F, Comuz J. Consequences of smoking for body weight, body fat distribution, and insulin resistance. Am J Clin Nutr 2008;87(4):801-809.

9. Task force of the European Society of Cardiology and the North American Society of Pacing and Electrophysiology. Heart rate variability. Standard of measurement, physical interpretation, and clinical use. Circulation 1996;93(5):1043-1065.

10. Kimura T, Matsumoto T, Akiyoshi M, Owa Y, Miyasaka N, Aso T, et al. Body fat and blood lipids in postmenopausal women are related to resting autonomic nervous system activity. Eur J Appl Physiol 2006;97(5):542-547.

11. Matsumoto T, Miyawaki T, Ue H, Zenji C, Moritani T. Autonomic responsiveness to acute cold exposure in obese and non-obese young women. Int J Obes 1999;23(8):793-800.

12. Matsumoto T, Miyawaki C, Ue H, Kanda T, Yoshitake Y, Moritani $\mathrm{T}$. Comparison of thermogenic sympathetic response to food intake between obese and non-obese young women. Obes Res 2001;9(2): 78-85.

13. Hukkanen J, Jacob P 3rd, Benowiz NL. Metabolism and disposition kinetics of nicotine. Pharmacol Rev 2005;57(1):79-115.

14. Benowitz NL, Gourlay SG. Cardiovascular toxicity of nicotine: implication for nicotine replacement therapy. J Am Coll Cardiol 1997; 29(7):1422-1231.

15. Amano M, Kanda T, Ue H, Moritani T. Exercise training and autonomic nervous system activity in obese individuals. Med Sci Sports Exercise 2001;33(8):1287-1291.

16. Nagai N, Sakane N, Hamada T, Kimura T, Moritani T. The effect of a high-carbohydrate meal on postprandial thermogenesis and sympathetic nervous system activity in boy with a recent onset of obesity. Metabolism 2005;54(4):430-438.
17. Shihara N, Yasuda K, Moritani T, Ue H, Uno M, Adachi T, et al. Synergistic effect of polymorphisms of uncoupling protein 1 and beta3-adrenergic receptor genes on autonomic nervous system activity. Int J Obes Relat Metab Disord 2001;25(6):761-766.

18. Nagai N, Sakane N, Ueno LM, Hamada T, Moritani T. The -3826 A-G variant of the uncoupling protein-1 gene diminishes postprandial thermogenesis after a high fat meal in healthy boys. J Clin Endocrinol Metab 2003;88(12):5661-5667

19. Brooks GA, Fahey TD, Baldwin KM. Obesity, body composition, and exercise. In: Exercise physiology - human bioenergetics and its application, 4th edition. New York: McGraw Hill; 2005:617-648.

20. Matsumoto T, Miyatsuji A, Miyawaki T, Yamagimoto Y, Moritani T. Potential association between leptin and sympatho-vagal activities in young obese Japanese women. Am J Hum Biol 2003;15(1):8-15.

21. Brooks GA, Fahey TD, Baldwin KM. Circulation and its control. In: Exercise physiology - human bioenergetics and its application, 4th edition. New York: McGraw Hill; 2005:312-362.

22. Collins LC, Cornelius MF, Vogal RL, Walker JF, Stamford BA. Effect of caffeine and/or cigarette smoking on resting energy expenditure. Int J Obes Relat Metab Disord 1994;18(8):551-556.

23. Perkins KA Epstein LH, DiMarco A, Fonte C, Stiller RL, Scierka A, et al. Acute thermogenic effects of nicotine combined with caffeine during light physical activity in male and female smokers. Am J Clin Nutr 1994;60(3):312-319.

24. Robinson S, York DA. The effect of cigarette smoking on the thermic response to feeding. Inter J Obes 1986;10(5):407-417.

25. Hofstetter A, Schutz Y, Jequler E, Wahren J. Increased 24-hour energy expenditure in cigarette smokers. N Engl J Med 1996;314(2): 79-82.

26. Audrain JE, Klesges RC, Klesges LM. Relationship between obesity and the metabolic effect of smoking in women. Health Psychol 1995; 14(2):116-123.

27. Minokoshi Y, Kim YB, Peroni OD, Fryer LGD, Muller C, Carling $\mathrm{D}$, et al. Leptin stimulates fatty-acid oxidation by activating AMPactivated protein kinase. Nature 2002;415(6869):339-343

28. Morse CI, Wurst RC, Jones DA, de Haan A, Degens H. Muscle fatigue resistance during stimulated contractions is reduced in young male smokers. Acta Physiol (Oxf) 2007;191(2):123-129.

29. Montes de Oca M, Loeb E, Torres SH, De Sanctis J, Hernándes N, Tálamo C. Peripheral muscle alterations in non-COPD smoker. Chest 2008;133(1):13-18.

30. Tokunaga K, Matsuzawa Y, Kotani K, Keno Y, Kobatake T, Fujioka $\mathrm{S}$, et al. Ideal body weight estimated from the body mass index with the lowest morbidity. Int J Obes 1991;15(1):1-5.

31. Thugane S, Sasaki S, Tsubono Y on behalf of the JPHC Study Group. Under- and overweight impact on mortality among middleaged Japanese men and women: a 10-y follow-up of JPHC study cohort I. Int J Obes Relat Metab Disord 2002;26(4):529-537.

32. Adamopoulos D, van de Borne P, Argacha JF. New insight into the sympathetic, endothelial and coronary effects of nicotine. Clin Exp Physiol 2008;35(4):458-463. 